\title{
An Expedient SERS Strip Tactic for Rapid On-Site Detection with Long-Time Sensitivity and Repeatability
}

\author{
Chen Wang $\mathbb{D}^{1},{ }^{1}$ Yueqian Wan, ${ }^{2}$ Yong Su, ${ }^{1}$ Yafei Cai, ${ }^{3}$ Shengjun Xiong $\mathbb{D}^{1},{ }^{1}$ Ding Yuan, \\ Zheng Xia, ${ }^{1}$ and Jie $\mathrm{Zhu}^{2}$ \\ ${ }^{1}$ HT-NOVA Co.,Ltd., Beijing 101312, China \\ ${ }^{2}$ State Key Laboratory for Advanced Metals and Materials, University of Science and Technology Beijing, Beijing 100083, China \\ ${ }^{3}$ College of Animal Science and Technology, Nanjing Agricultural University, Nanjing 210095, China
}

Correspondence should be addressed to Shengjun Xiong; xiong.shengjun@htnova.com

Received 14 January 2021; Revised 24 March 2021; Accepted 12 April 2021; Published 20 April 2021

Academic Editor: Lingxue Kong

Copyright (c) 2021 Chen Wang et al. This is an open access article distributed under the Creative Commons Attribution License, which permits unrestricted use, distribution, and reproduction in any medium, provided the original work is properly cited.

Surface-enhanced Raman spectroscopy (SERS) has attracted lots of interest in academic and industrial fields in recent years. The improvement in long-time sensitivity and repeatability is highly demanded by the on-site applications. Herein, we present an expedient SERS strip tactic with these desired advantages. Specifically, the tactic utilized the outstanding stability of colloidal particles to maintain the SERS materials during the storage. Upon usage, the strip is rapidly prepared on-site, and then the targets were sampled with a dip-coating and heating method, which is designed to standardize the whole detection process with the sensitivity kept. Thanks to the tactic, only one-third of SERS sensitivity decay was observed for rhodamine 6G after half a year. Besides rhodamine $6 \mathrm{G}$, the SERS spectra of different animal blood samples were also investigated with the SERS strip tactic, and a species-based discrimination capability was preliminarily demonstrated.

\section{Introduction}

Raman spectroscopy is a kind of fingerprint spectra resulting from the vibration of chemical bonds, which could be used to identify the molecules and materials [1]. The measurement of Raman spectra is noninvasive, fast, and resistant to water interference. Additionally, the applications of surfaceenhanced Raman spectroscopy (SERS) have extended to trace detection fields [1-5], such as the sniffing of explosives [6], drugs [7], pesticide residues [8], prohibited food additions [9], and microorganism identifications $[10,11]$. With the development of hand-held Raman spectrometers, the detection method has demonstrated great potential for onsite trace detection and identification, and therefore, the sensing materials with long-term sensitivity, repeatability, and low cost become highly desired [12].

The amplification of electromagnetic fields generated by the excitation of localized surface plasma is the source of SERS and roughened surface could enhance the amplification and the adsorption of target molecules [13]. Chemical synthesized colloidal nanoparticles (NPs) of Ag and Au are the major source of SERS materials due to their low cost, long lifetime, and high sensitivity [14]. However, the issues on repeatability and inconvenient operations may still hinder the SERS applications. For example, due to ions, temperature, and organic solvents, the colloidal is easy to aggregate quickly after the samples are transferred in to the colloids, causing low uniformity of the samples and consequential uncertainty of the measurements [15]. Additionally, the use of colloidal NPs requires precise liquid transferring operation in microliter levels. To achieve expedient usage, better sensitivity, and repeatability, the paperbased SERS strips fabricated with printed NPs were explored, and the NPs on the strips were closely stacked on the substrates [16]. Due to the high surface energy and the lack of protection of solvents, the Ostwald ripening of the NPs, and the reaction between NPs with oxygen, humidity and even paper substrates can gradually lower the sensitivity of the conventional SERS strips, leading to a short lifetime in days.

To increase the ease of operations, the repeatability of colloidal NPs, and the short lifetime of conventional SERS 
strips, herein we introduce a hybrid tactic to improve the long-time sensitivity and repeatability of SERS detection with simple operations as shown in Scheme 1, which is suitable for rapid on-site applications [17]. In detail, chemically synthesized colloidal AgNPs were prepared and maintained for the low cost and great storage stability. Upon usage, sensing strips were fabricated through the direct dipcoating of colloidal NPs onto the blank paper, followed by rapid heating of a microheater. During target sampling, the as-prepared strip was directly dipped into the sample solution and then heated with the same microheater to accelerate the operation and to avoid the "coffee ring" effect $[18,19]$. Therefore, in a single detection, the strip was always freshly fabricated from the stable colloidal NPs to ensure the long-time sensitivity, and the whole process was standardized with dipping and heating operations for better uniformity and repeatability without any liquid transferring operations and unexpected aggregation of NPs. Additionally, the strip fabrication step and target sampling step could be repeated several times to increase the overall sensitivity of the strips. With this SERS strip tactic, the spectra of rhodamine 6G (Rh6G) solution and diluted blood samples from different animals were studied with a hand-held Raman spectrometer to mimic the on-site application scenes. The Raman spectra of Rh6G and blood samples were clearly observed, and the blood samples could be further partially discriminated based on the animal species. This proof-ofconcept work may demonstrate a potential application of the SERS technique in forensic and wild animal protection fields [20].

\section{Materials and Methods}

2.1. Preparation of Colloidal AgNPs. Colloidal AgNPs were prepared following the similar method of previous literature [16]. To increase the concentration of AgNPs, the concentrations of the precursors were tripled compared to the original method. In a typical preparation, $90 \mathrm{mg}$ of $\mathrm{AgNO}_{3}$ was added to $160 \mathrm{ml}$ ultrapure water and then heated in a round-bottom flask with $120^{\circ} \mathrm{C}$ with vigorous stirring for 10 minutes. Then, $10 \mathrm{ml}$ of a solution containing $100 \mathrm{mg}$ of sodium citrate was added to the flask dropwise within 5 minutes. After half an hour, the solution turned brown grey and was removed from the heating bath to cool down to room temperature.

\subsection{General Detection Operations of the SERS Strip Tactic.} The SERS strip tactic for on-site applications requires a microheater containing a precise closed-loop temperature control plate, blank strips made from Fisherbrand pure cellulose chromatography paper, vials containing the prepared colloidal AgNPs, empty vials for liquid samples, and a hand-held Raman spectrometer. And the general detection operations include three steps. The first step is the fabrication of a test strip. The temperature of the microheater was optimized to $65{ }^{\circ} \mathrm{C}$ for strip fabrication. A blank strip $(\sim 4 \mathrm{~cm} \times 0.5 \mathrm{~cm})$ was directly dipped into the colloidal AgNPs vial for 5 seconds and then was quickly pulled out with a following baking for 1 minute with the microheater. The first step could be performed a few times to enhance the detection sensitivity. The second step is the sample preparation and transferring. The target molecule or material should be in a liquid state itself or dissolved in a certain kind of solvent to form a homogeneous state, which is suitable for strip dipping. The insoluble materials should be filtered before sampling to avoid possible interference left on the strip surface. Then, a fabricated strip is dipped to the sample vial for 5 seconds, which contains the solution of target samples. Similar to the first step, the strip is taken out and baked for 1 minute with the microheater, and this process could also be done for multiple times to accumulate the target molecules within the strip. The temperature of the microheater should be optimized for different samples. For Rh6G and blood samples, the temperature was kept as $65^{\circ} \mathrm{C}$. The third step is the spectrum measurement. The freshly prepared strip with target molecules is directly measured with a hand-held Raman spectrometer. The central area of a strip is recommended to avoid the edge effect of the strip. All the blood samples were kept in $-20^{\circ} \mathrm{C}$ refrigerator and contained trisodium citrate as the anticoagulant. Before sampling, they were diluted by 10 times with $0.9 \% \mathrm{NaCl}$ water solution. In the experiments, the strips were dipcoated and heated for three times, and the blood samples were dipped and heated for once.

2.3. Morphological and Spectral Characterizations. The Raman spectra were characterized with a hand-held Raman spectrometer (model CR-2000, HT-NOVA Co., Ltd.) in all the experiments. The excitation laser is $785 \mathrm{~nm}$, and the detectable wavelength ranges $200 \sim 3200 \mathrm{~cm}^{-1}$ with the spectral resolution ranging $4 \sim 6 \mathrm{~cm}^{-1}$. Morphological observations were conducted by scanning electron microscopy (SEM, Carl Zeiss, Supra 55) and transmission electron microscope (TEM, FEI, Tecnai F30).

\section{Results and Discussion}

3.1. Preparation of Test Strips and Morphological Characterizations. Figure 1(a) exhibits the microheater designed for the SERS strip tactic. The heating zone consisted of a closed-loop controlled heater targeting $65^{\circ} \mathrm{C}$ and a hollow cover to allow the escape of water vapor generated during the baking. The fast evaporation of the solvent could restrain the "coffee ring" effect, which is a common reason for heterogeneity in the wet coating process. The prepared AgNPs had good dispersion in the colloidal solution, ensuring the uniformity of dip-coating of the strips. To begin the detection, the blank test strip was dipped into the vial containing the colloidal AgNPs for 5 seconds, as shown in Figure 1(b). Then, the strip was placed in the microheater to fabricate the test strip rapidly. After the baking, the color of the strip with AgNPs appeared to be light grey. As a control, the blank test strip was also baked following the same procedure, while the color remained white, indicating the color change should be due to the adhesion of AgNPs onto the paper. When the first cycle of dip-coating and heating 


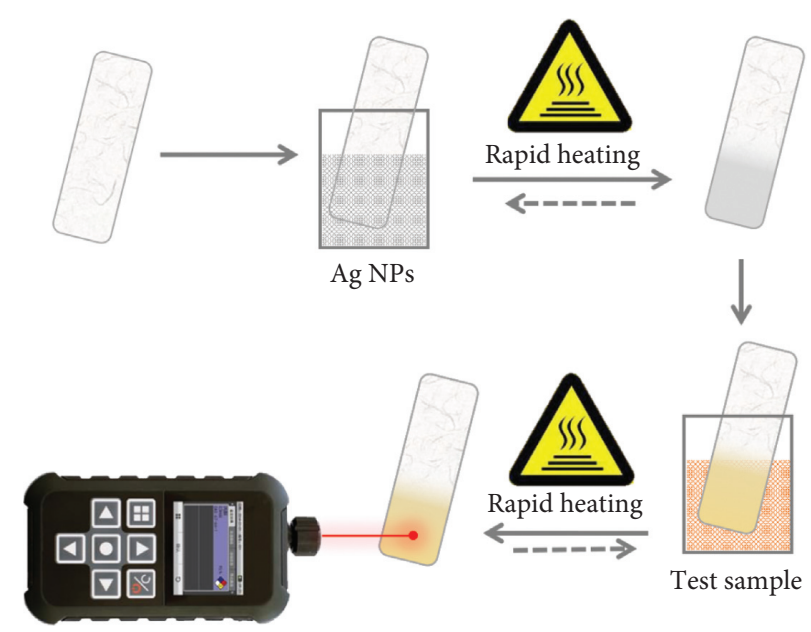

Scheme 1: The general detection operations of the SERS strip tactic.

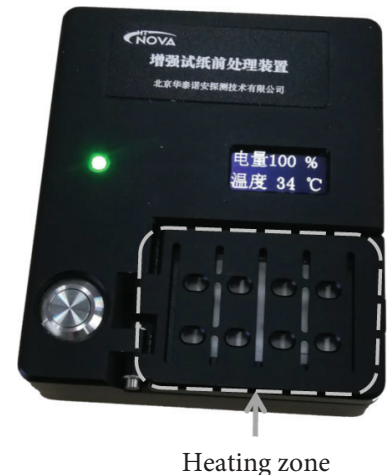

(a)

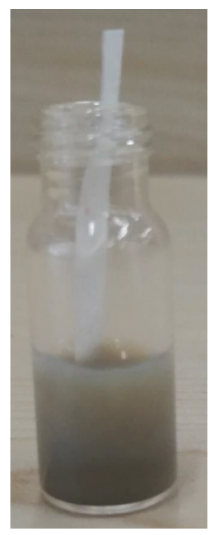

(b)

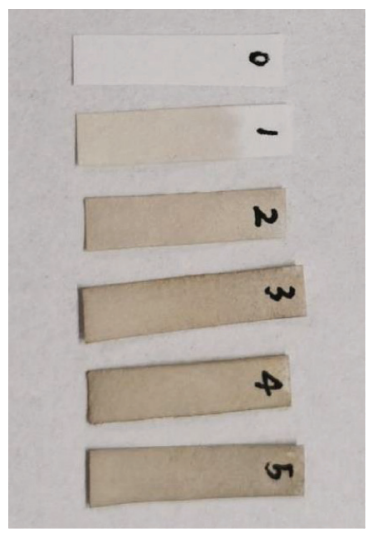

(c)

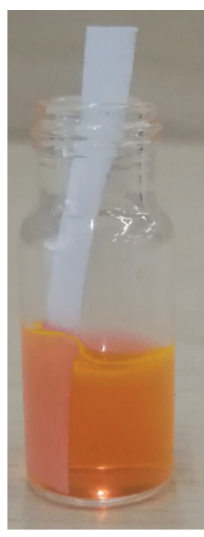

(d)

Figure 1: (a) The photo of the microheater, (b) the dip-coating of a test strip into colloidal AgNPs, (c) the prepared test strips with different cycles of dip-coating and heating, and (d) the target sampling of Rh6G solution with a prepared test strip.

was done, there is an option to repeat the fabrication to further enrich the AgNPs on the surface. Figure 1(c) displays the strips with different cycles of fabrications ranging from zero to five. Correspondingly, the color of these strips becomes darker and darker, demonstrating the net accumulation of AgNPs on the surface in each fabrication step. When the SERS strips were prepared, they were ready for the target sampling step to contact the liquid sample solutions. Figure 1(d) displays this step for Rh6G water solution, which is quite similar to the strip fabrication step, and this sampling step is also accumulable for better overall sensitivity if necessary.

To confirm the coherence between the color change of test strips and the accumulation of AgNPs and to further study the AgNPs distribution on the strips, the morphology of colloidal AgNPs and the fabricated strips were characterized with TEM and SEM, respectively. Figure 2(a) shows a typical TEM image of AgNPs. The shape of the AgNPs was between spheres and polyhedrons with multiple facets. Their diameters ranged from $35 \mathrm{~nm}$ to $50 \mathrm{~nm}$ with a narrow distribution. The surface of these AgNPs was clear and sharp. After the fabrication of strips, the central part of the strip was cut for SEM characterization to ensure representativeness. Figures 2(b) 2(f) show the surface status of the strips with 1 5 cycles of dip-coating and heating, and Figures S1(a) S1(e) also confirm the similar morphological status in larger areas, respectively. After the first cycle, the AgNPs were clearly found on the test strips and formed small clusters containing tens of AgNPs, instead of evenly distributed across the paper interface. Such cluster-like structures may indicate that the interaction between the AgNPs themselves is stronger than the interaction between the AgNPs and paper. So, even with a low amount of AgNPs contact, the aggregation could also be achieved efficiently. After the second cycle, a few small clusters "grew" to tiny islands and more clusters formed, indicating the accumulation was preferred around existing AgNPs with the favourable interaction. Then, in the following cycles, the islands tended to continue growing into large areas, and some clusters started to merge into large islands. After the fifth cycle, the large islands containing a large amount of AgNPs became dominant on the surface of the paper. Therefore, the SEM images may support the positive correlation between the color change and the 


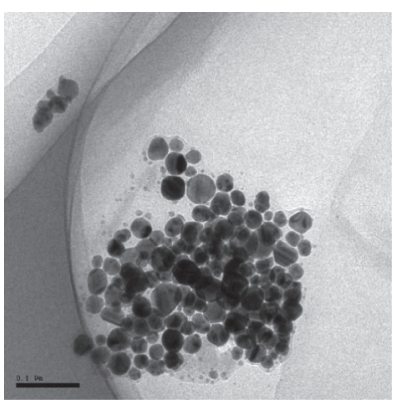

(a)

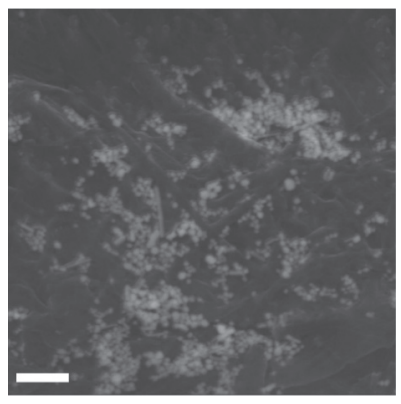

(d)

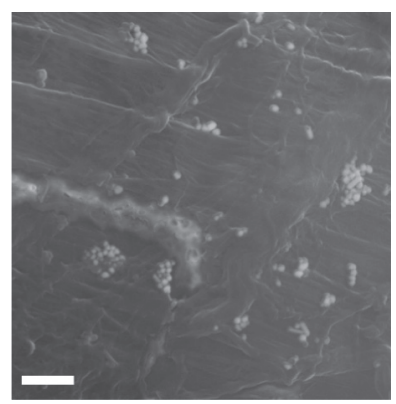

(b)

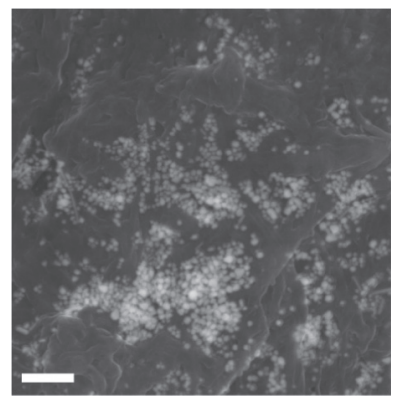

(e)

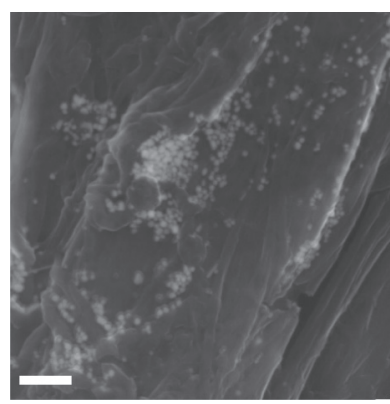

(c)

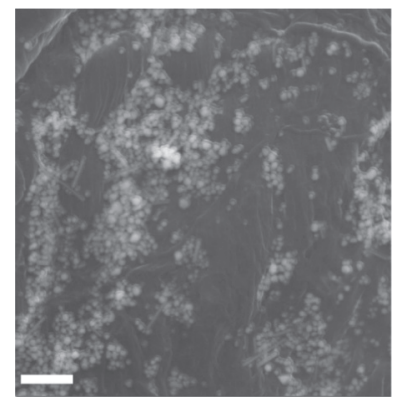

(f)

Figure 2: (a) The TEM image of AgNPs and the SEM images of test strips surface after (b) 1 cycle, (c) 2 cycles, (d) 3 cycles, (e) 4 cycles, and (f) 5 cycles of dip-coating and heating. The scale bar of the TEM image is $0.1 \mu \mathrm{m}$, and the scale bar of the SEM images is $0.5 \mu \mathrm{m}$.

amount of AgNPs. Additionally, it was clear that, even with once dip-coating and heating, the AgNPs aggregation, or "hot-spots," efficiently formed and should be capable of offering SERS sensitivity. More fabrication cycles would cause consequent aggregations so that the sensitivity of the strip was also expected to increase.

3.2. SERS Effect of Rh6G Based on the Strip Tactic. According to the morphological study, the strip tactic implied great potential on the SERS applications with tunable sensitivity. To quantitatively evaluate the performance of the fabricated strips, several series of SERS spectra of Rh6G water solution were measured. Figure 3(a) show the first series of SERS spectra of different concentrations of Rh6G sampled by the test strips with three fabrication cycles. The concentration range of Rh6G was from $2 \times 10^{-4} \mathrm{mM}$ to $0.1 \mathrm{mM}$, and except for the spectra of $2 \times 10^{-4} \mathrm{mM}$ of Rh6G, the spectra from other concentrations showed clear peaks of Rh6G. In Figure S2, multiple spectra of a $5 \times 10^{-3} \mathrm{mM}$ Rh6G solution from different strips confirmed the good reproducibility of the tactic. And for different concentrations, from the low Raman shift side, the six strongest peaks were also identical, located at $612 \mathrm{~cm}^{-1}, 771 \mathrm{~cm}^{-1}, 1183 \mathrm{~cm}^{-1}$, $1312 \mathrm{~cm}^{-1}, 1364 \mathrm{~cm}^{-1}$, and $1510 \mathrm{~cm}^{-1}$, and they were consistent with previous literature, indicating the heating temperature was suitable for Rh6G [21]. Since the spectra of the Rh6G were similar, the peak intensities (with baseline correction) at $1364 \mathrm{~cm}^{-1}$ were calculated and plotted in Figure 3(b). A clear positive correlation relationship between the concentrations and selected peak intensity was found, indicating the qualitative and quantitative capability of the strip tactic.
In the second series, the adjustable sensitivity via the number of fabrication cycles was studied. The strips with fabrication cycles from one to five were prepared to measure the SERS spectra of $0.6 \mathrm{mM}$ of Rh6G solution, as shown in Figure 3(c). The results demonstrated that, even with only 1 cycle of fabrication, the strip was sensitive enough to provide the SERS spectrum with a high signalto-noise ratio, which was consistent with the surface AgNPs clusters found in the morphological study of the strips with one cycle of fabrication. Indeed, with the increase of the fabrication cycles, all the characteristic peaks of Rh6G grew gradually, indicating that more AgNPs could enhance the SERS signals. And this effect was also observed for different concentrations of Rh6G, as shown in Figure S3. According to the peak intensity (with baseline correction) at $1364 \mathrm{~cm}^{-1}$, with the same concentration and measurement configuration of the Raman spectrometer, the intensity tunable range contributed by the fabrication cycles was 4-5 times, as shown in Figure 3(d). It was interesting to note that the enchantment ratio from the $2^{\text {nd }}$ to the $4^{\text {th }}$ cycle was relatively higher than the number from the $1^{\text {st }}$ to the $2^{\text {nd }}$ cycle and from the $4^{\text {th }}$ to the $5^{\text {th }}$ cycle. Considering the morphological study, the stages between the $2^{\text {nd }}$ and the $4^{\text {th }}$ cycles could well correspond to the formation of larger islands of AgNPs on the surface. While in the $1^{\text {st }}$ cycle, only small clusters formed and in the $5^{\text {th }}$ cycle, the net accumulation of islands may be limited by dissolve equilibrium. Additionally, combining the flexible dipping times of the sampling step, the total tunable sensitivity windows of this method were over twenty times. Considering the concentrations of target molecules for on-site measurement are usually unknown and enriching target molecules requires mass of work and 


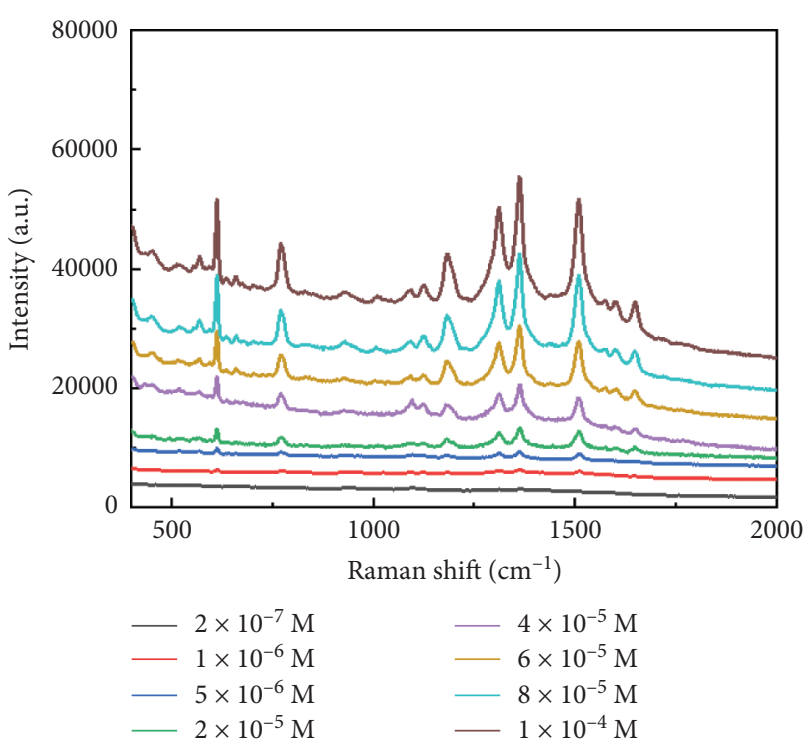

(a)

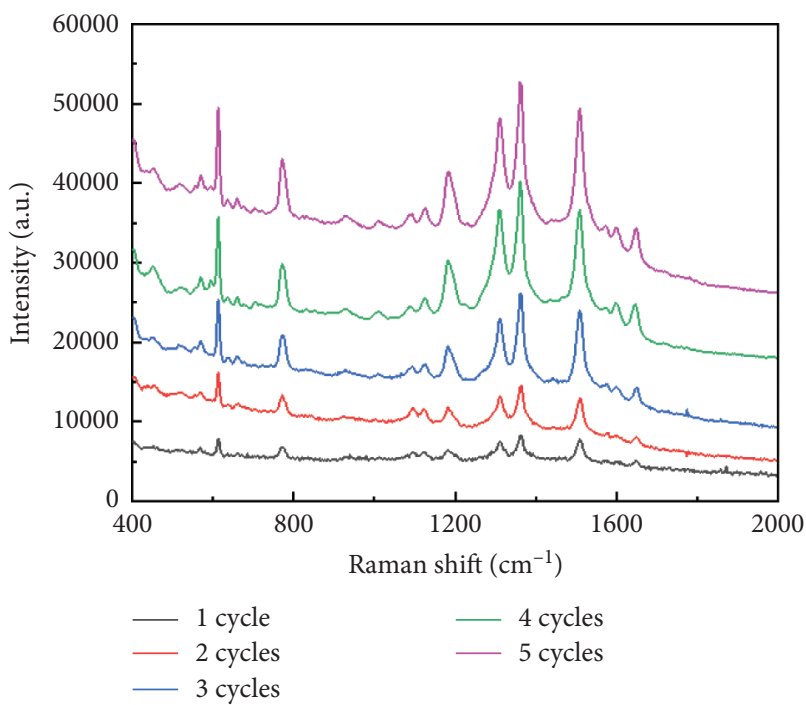

(c)

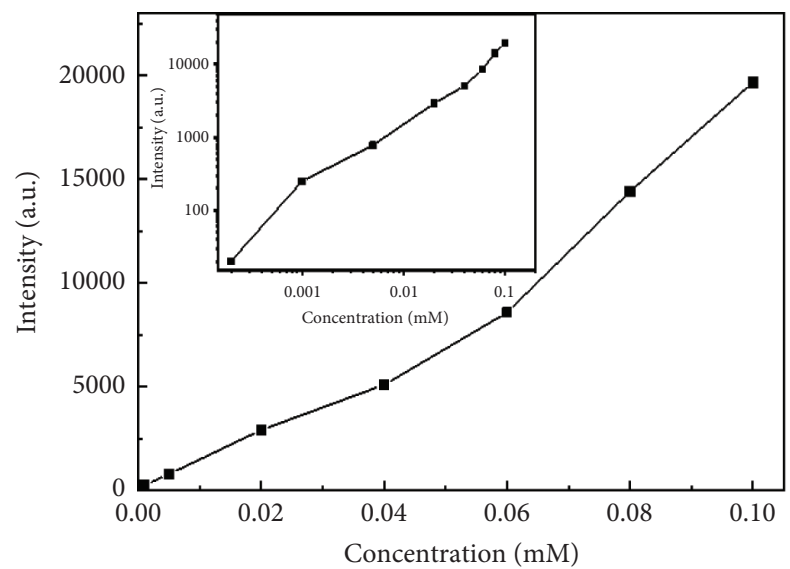

(b)

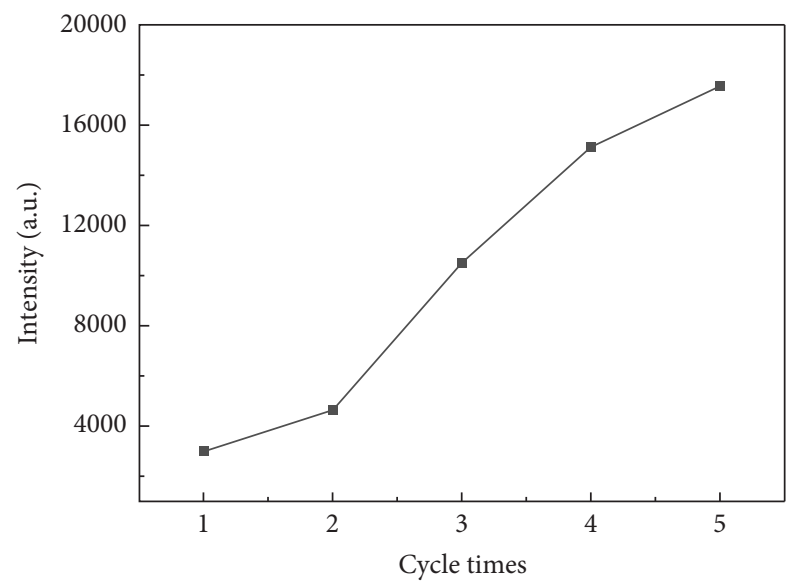

(d)

FIGURE 3: (a) The SERS spectra and (b) the $1364 \mathrm{~cm}^{-1}$ peak intensity plot of different concentrations of Rh6G sampled by test strips with 3 cycles of fabrication and (c) the SERS spectra and (d) the $1364 \mathrm{~cm}^{-1}$ peak intensity plot of $0.6 \mathrm{mM}$ of Rh6G sampled by test strips with different fabrication cycles.

additional instruments, such unique sensitivity flexibility may greatly assist in the on-site detections for different targets and applications.

3.3. Stability of the SERS Strip Tactic. As a practical tactic for on-site detection, besides the sensitivity and ease of use, the long-term stability on performance is essential, especially for quantitative or semiquantitative applications. According to previous literature, the colloid system is relatively stable if stored in a controlled environment, while the paper-based predeposited strips meet the fast decay of sensitivity [16]. This fact is one of the motivations of the tactic that the NPs are stored in the colloidal state and then freshly are prepared into strips on the field with a rapid and controllable microheater.
To prove the advantage of this approach, a series of long-time tests of Rh6G spectra with predeposited strips and on-site fabricated strips were conducted with the same batch of colloidal AgNPs. Half of the NPs were used to fabricate strips at the beginning of the experiments with the dip-coating and heating method. These strips were counted as the predeposited strips and immediately packed with an aluminium film plastic bag under vacuum. And the other half of the colloidal AgNPs was kept in vials for the SERS strip tactic. All the strips and colloids were kept at $4^{\circ} \mathrm{C}$ temperature and in dark. Then, in every 2 days, $0.5 \mathrm{mM}$ of Rh6G water solution was measured with the sealed predeposited strips and the freshly fabricated strips with the stored colloidal AgNPs. After ten days, the measured spectra were compared in Figure 4(a). It was clear that the sensitivity of the predeposited strips 


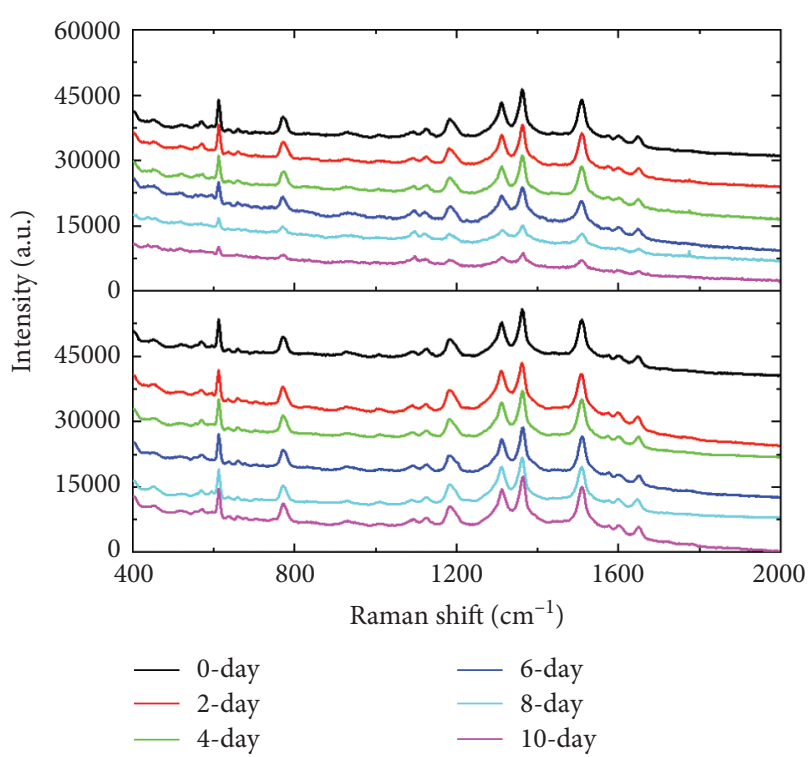

(a)

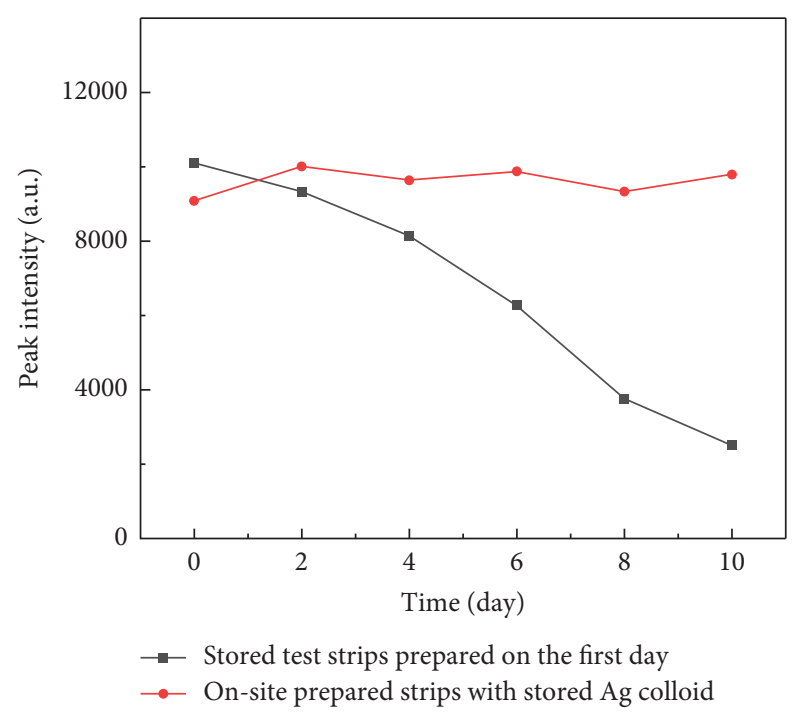

(b)

Figure 4: (a) The Raman spectra of $0.5 \mathrm{mM}$ of Rh6G sampled by sealed predeposited strips (up) and strips fabricated with the tactic (down) and (b) the $1364 \mathrm{~cm}^{-1}$ peak intensity plot of these spectra in ten days.

decreased significantly though they were carefully sealed, while the sensitivity of the strips fabricated the tactic was almost kept based on the $1364 \mathrm{~cm}^{-1}$ peak intensity plot, as shown in Figure 4(b). Then, in the subsequent experiments, the predeposited strips could barely detect Rh6G solution after one month, while with the strip tactic, the peak intensity of Rh6G spectra only suffers about one-third loss after six months, compared to the initial intensity. Consequently, such on-site fabrication tactic was confirmed to help prevent the sensitivity decay with little sacrifice on strip fabrication time. The advantage on long-term stability, in addition to the tunable sensitivity, could provide a convenient tool for on-site applications.

3.4. Detection and Discrimination of Animal Blood Samples. With the confirmation of the sensitivity, long-term stability, and repeatability, the SERS strip tactic was applied to study a series of blood samples, since rapid blood identification is usually desired in forensic and wild animal protection fields, and some previous literature has reported that Raman spectra could be used for the detection and discrimination of blood [22-24]. Although heating would change the biological activity of blood samples, the heating temperature was still set to $65^{\circ} \mathrm{C}$ because the Raman spectroscopy mainly reveals the information of primary chemical bonds, instead of the folding structures of biomacromolecules. The blood samples from seven species of animals were measured following the general operations for 30 times per species, and all the spectra are demonstrated in Figure S4. The high similarity of the spectra from a certain species indicated the good reproducibility of the strip tactic. Figure 5 shows the average Raman spectra, and compared to the blank test strips, the blood samples showed several specific peaks, such as the peaks at $480 \mathrm{~cm}^{-1}, 660 \mathrm{~cm}^{-1}, 1128 \mathrm{~cm}^{-1}, 1221 \mathrm{~cm}^{-1}$,

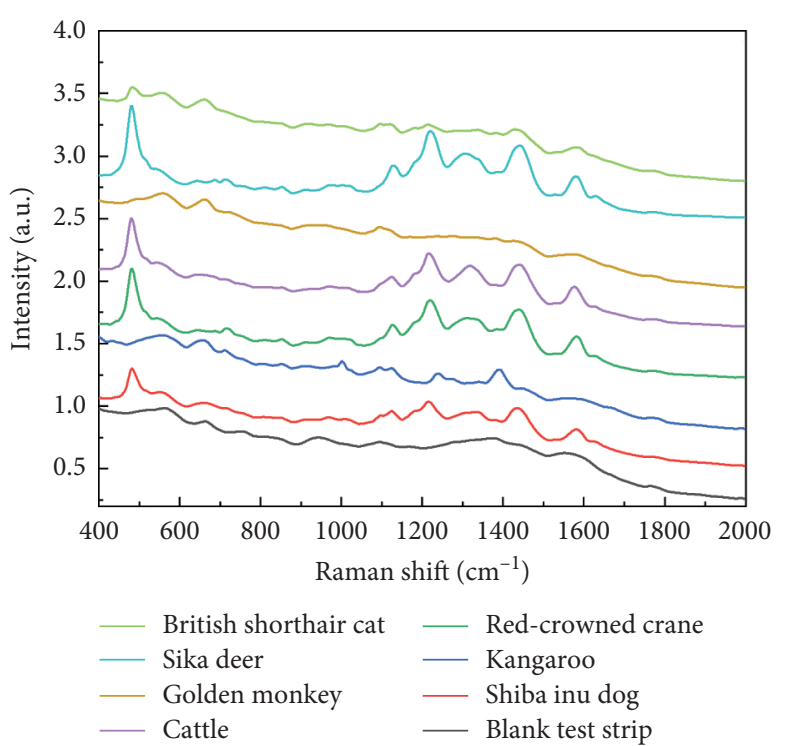

FIgURE 5: The average Raman spectra of blood samples from seven species of animals.

$1315 \mathrm{~cm}^{-1}, 1439 \mathrm{~cm}^{-1}$, and $1578 \mathrm{~cm}^{-1}$, which were also observed in other literature $[25,26]$. Specifically, $660 \mathrm{~cm}^{-1}$ and $1221 \mathrm{~cm}^{-1}$ are related to haemoglobin in the blood; $1128 \mathrm{~cm}^{-1}$ and $1315 \mathrm{~cm}^{-1}$ may be from the spermine phosphate hexahydrate; and $1439 \mathrm{~cm}^{-1}$ closely matches the Raman bands of serum albumin [27, 28].

However, the blood components of large animals are complex, so the characteristic peaks based interpretation into chemical bonds and molecules may be complicated. The identification goal is not only the chemical components but also the species information. Therefore, in a lot of cases, the classification algorithms towards final labels, instead of 


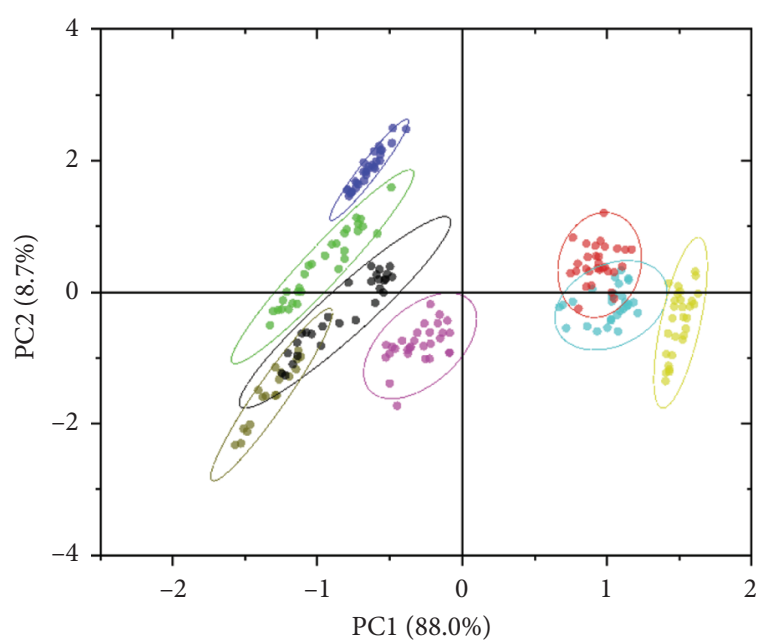

$\begin{array}{ll}\text { - British shorthair cat } & \text { - Red-crowned crane } \\ \text { - Cattle } & \text { - Shiba inu dog } \\ \text { - Golden monkey } & \text { - Sika deer } \\ \text { - Kangaroo } & \text { - Blank test strip }\end{array}$

Figure 6: The PCA results of the Raman spectra of seven species of animal blood samples with $95 \%$ confident ranges.

matching the characteristic peaks, were more frequently applied. Such algorithms mainly require good reproducibility and consistency between the labelled samples and the detected unknown samples and are relatively less influenced by the poetical chemical or physical change during the tests if the same change happens in both the labelled samples and the detected unknown samples. Herein, we applied the principle component analysis (PCA), which was frequently applied for the classification goals [29], to these spectra to attempt to cluster the spectra based on species. Overall, the first two components covered over $95 \%$ of the information of the original spectra, indicating the feasibility of the PCA method to these spectra. Figure 6 shows the PCA clustering analysis with a $95 \%$ confident range of each cluster. From the overlaps of different species, the SERS signals from the strips could fully discriminate 5 species from each other, such as kangaroo, dog, golden monkey, and sika deer. On the other hand, a part of the spectra of cat blood samples overlapped with the blank strips, which may be caused by relatively weak signals of these spectra. The confident ellipses of cattle and red-crowned crane SERS spectra shared about one-third of samples, which may cause less accurate prediction regarding these two animals. To further verify the classification potential, the third principle component, which carried about $1.5 \%$ of the information, was also considered. Under this condition, the spectra from the cat could successfully separate from the blank strips under $95 \%$ confidence while cattle and redcrowned crane still cannot be fully distinguished with the SERS strips and the hand-held Raman spectrometer. In summary, in the preliminary experiments, the animal species could be probably discriminated via the Raman spectra of blood under $95 \%$ confidence. If the goal is only yes/no binary classification towards a certain kind of species, the true positive rate should be higher.

\section{Conclusions}

In this article, we present a SERS strip tactic which has great potential for on-site measurement with long-term sensitivity and repeatability. With a dedicated microheater, the SERS strips could be fabricated easily and efficiently on the field. In addition, the dip-coating and rapid heating combination method could also be applied to the target sampling process, which not only increases the uniformity and detection efficiency but also enables the tunable window of the sensitivity of the strip tactic about twenty times. Such flexibility may provide great help for the real-world detection when the concentration of the target is unknown or out of the default range. To further demonstrate the practical potential of the strip tactic, the blood samples from different animal species were measured with the SERS strip tactic following the general operations. With the SERS strips, the Raman spectra from all the samples were observed, and by applying the PCA algorithm to those spectra, the spectra were partially discriminated. Such promising results indicated the SERS strip tactic may contribute to forensic, wild animal protection and many other fields, where the on-site expedient detection and screening solutions are desired.

\section{Data Availability}

The data in the manuscript are obtained through experiments. If necessary, the original data can be provided by the authors (please contact xiong.shengjun@htnova.com) to ensure the repeatability of the experiment.

\section{Conflicts of Interest}

The authors declare that there are no conflicts of interest regarding the publication of this paper.

\section{Acknowledgments}

This work was supported by the fund from the National Key R\&D Program of China (2018YFC1200203).

\section{Supplementary Materials}

Figure S1: the large-area SEM images of test strips surface. Figure S2: the reproducibility test of Rh6G solution. Figure S3: the parallel data of different concentrations of Rh6G with strips from different cycles of fabrication. Figure S4: the spectra summary of different animal blood samples. (Supplementary Materials)

\section{References}

[1] P. L. Stiles, J. A. Dieringer, N. C. Shah, and R. P. Van Duyne, "Surface-enhanced Raman spectroscopy," Annual Review of Analytical Chemistry, vol. 1, no. 1, pp. 601-626, 2008.

[2] E. C. Le Ru, E. Blackie, M. Meyer, and P. G. Etchegoin, "Surface enhanced Raman scattering enhancement factors: a comprehensive study," The Journal of Physical Chemistry C, vol. 111, no. 37, pp. 13794-13803, 2007.

[3] E. J. Blackie, E. C. Le Ru, and P. G. Etchegoin, "Single-molecule surface-enhanced Raman spectroscopy of nonresonant 
molecules," Journal of the American Chemical Society, vol. 131, no. 40, pp. 14466-14472, 2009.

[4] S. Nie and S. R. Emory, "Probing single molecules and single nanoparticles by surface-enhanced Raman scattering," Science, vol. 275, no. 5303, pp. 1102-1106, 1997.

[5] E. C. Le Ru, M. Meyer, and P. G. Etchegoin, "Proof of singlemolecule sensitivity in surface enhanced Raman scattering (SERS) by means of a two-analyte technique," The Journal of Physical Chemistry B, vol. 110, no. 4, pp. 1944-1948, 2006.

[6] T. Demeritte, R. Kanchanapally, Z. Fan et al., "Highly efficient SERS substrate for direct detection of explosive TNT using popcorn-shaped gold nanoparticle-functionalized SWCNT hybrid," The Analyst, vol. 137, no. 21, pp. 5041-5045, 2012.

[7] J. Song, J. Zhou, and H. Duan, "Self-assembled plasmonic vesicles of SERS-encoded amphiphilic gold nanoparticles for cancer cell targeting and traceable intracellular drug delivery," Journal of the American Chemical Society, vol. 134, no. 32, pp. 13458-13469, 2012.

[8] S. Sánchez-Cortés, C. Domingo, J. V. García-Ramos, and J. A. Aznárez, "Surface-enhanced vibrational study (SEIR and SERS) of dithiocarbamate pesticides on gold films," Langmuir, vol. 17, no. 4, pp. 1157-1162, 2001.

[9] A. P. Craig, A. S. Franca, and J. Irudayaraj, "Surface-enhanced Raman spectroscopy applied to food safety," Annual Review of Food Science and Technology, vol. 4, no. 1, pp. 369-380, 2013.

[10] S. Shanmukh, L. Jones, Y.-P. Zhao, J. D. Driskell, R. A. Tripp, and R. A. Dluhy, "Identification and classification of respiratory syncytial virus (RSV) strains by surface-enhanced Raman spectroscopy and multivariate statistical techniques," Analytical and Bioanalytical Chemistry, vol. 390, no. 6, pp. 1551-1555, 2008.

[11] R. M. Jarvis and R. Goodacre, "Characterisation and identification of bacteria using SERS," Chemical Society Reviews, vol. 37, no. 5, pp. 931-936, 2008.

[12] C. Muehlethaler, M. Leona, and J. R. Lombardi, "Review of surface enhanced Raman scattering applications in forensic science," Analytical Chemistry, vol. 88, no. 1, pp. 152-169, 2016.

[13] S.-Y. Ding, E.-M. You, Z.-Q. Tian, and M. Moskovits, "Electromagnetic theories of surface-enhanced Raman spectroscopy," Chemical Society Reviews, vol. 46, no. 13, pp. 4042-4076, 2017.

[14] M. Fan, G. F. S. Andrade, and A. G. Brolo, "A review on recent advances in the applications of surface-enhanced Raman scattering in analytical chemistry," Analytica Chimica Acta, vol. 1097, pp. 1-29, 2020.

[15] H. Wei, M. R. Willner, L. C. Marr, and P. J. Vikesland, "Highly stable SERS $\mathrm{pH}$ nanoprobes produced by co-solvent controlled AuNP aggregation," The Analyst, vol. 141, no. 17, pp. 5159-5169, 2016.

[16] W. W. Yu and I. M. White, "Inkjet printed surface enhanced Raman spectroscopy array on cellulose paper," Analytical Chemistry, vol. 82, no. 23, pp. 9626-9630, 2010.

[17] J. Sun, L. Gong, W. Wang, Z. Gong, D. Wang, and M. Fan, "Surface-enhanced Raman spectroscopy for on-site analysis: a review of recent developments," Luminescence, vol. 35, no. 6 , pp. 808-820, 2020.

[18] D. Mampallil and H. B. Eral, "A review on suppression and utilization of the coffee-ring effect," Advances in Colloid and Interface Science, vol. 252, pp. 38-54, 2018.

[19] S. Das, A. Dey, G. Reddy, and D. D. Sarma, "Suppression of the coffee-ring effect and evaporation-driven disorder to order transition in colloidal droplets," The Journal of Physical Chemistry Letters, vol. 8, no. 19, pp. 4704-4709, 2017.
[20] A. Sikirzhytskaya, V. Sikirzhytski, G. McLaughlin, and I. K. Lednev, "Forensic identification of blood in the presence of contaminations using Raman microspectroscopy coupled with advanced statistics: effect of sand, dust, and soil," Journal of Forensic Sciences, vol. 58, no. 5, pp. 1141-1148, 2013.

[21] A. M. Michaels, M. Nirmal, and L. E. Brus, "Surface enhanced Raman spectroscopy of individual rhodamine 6G molecules on large Ag nanocrystals," Journal of the American Chemical Society, vol. 121, no. 43, pp. 9932-9939, 1999.

[22] D. Drescher, T. Büchner, D. McNaughton, and J. Kneipp, "SERS reveals the specific interaction of silver and gold nanoparticles with hemoglobin and red blood cell components," Physical Chemistry Chemical Physics, vol. 15, no. 15, pp. 5364-5373, 2013.

[23] S. Feng, R. Chen, J. Lin et al., "Nasopharyngeal cancer detection based on blood plasma surface-enhanced Raman spectroscopy and multivariate analysis," Biosensors and Bioelectronics, vol. 25, no. 11, pp. 2414-2419, 2010.

[24] R. A. Alvarez-Puebla and L. M. Liz-Marzán, "SERS-based diagnosis and biodetection," Small, vol. 6, no. 5, pp. 604-610, 2010.

[25] A. Sikirzhytskaya, V. Sikirzhytski, and I. K. Lednev, "Determining gender by Raman spectroscopy of a bloodstain," Analytical Chemistry, vol. 89, no. 3, pp. 1486-1492, 2017.

[26] K. C. Doty, G. McLaughlin, and I. K. Lednev, "A Raman "spectroscopic clock" for bloodstain age determination: the first week after deposition," Analytical and Bioanalytical Chemistry, vol. 408, no. 15, pp. 3993-4001, 2016.

[27] V. Sikirzhytski, K. Virkler, and I. K. Lednev, "Discriminant analysis of Raman spectra for body fluid identification for forensic purposes," Sensors, vol. 10, no. 4, pp. 2869-2884, 2010.

[28] B. R. Wood and D. McNaughton, "Raman excitation wavelength investigation of single red blood cellsin vivo," Journal of Raman Spectroscopy, vol. 33, no. 7, pp. 517-523, 2002.

[29] H. Shin, H. Jeong, J. Park, S. Hong, and Y. Choi, "Correlation between cancerous exosomes and protein markers based on surface-enhanced Raman spectroscopy (SERS) and principal component analysis (PCA)," ACS Sensors, vol. 3, no. 12, pp. 2637-2643, 2018. 